\title{
Polyfunctional inorganic-organic hybrid materials: an unusual kind of NLO active layered mixed metal oxalates with tunable magnetic properties and very large second harmonic generation
}

Elena Cariati, ${ }^{\text {a* }}$ Roberto Macchi, ${ }^{a}$ Dominique Roberto, ${ }^{a}$ Renato Ugo ${ }^{a}$, Simona Galli, ${ }^{b}$ Nicola Casati, ${ }^{c}$ Piero Macchi, ${ }^{*}$ Angelo Sironi, ${ }^{\circ}$ Lapo Bogani, ${ }^{\mathrm{d}}$ Andrea Caneschi ${ }^{\mathrm{d}^{*}}$ and Dante Gatteschi ${ }^{\mathrm{d}^{*}}$

${ }^{a}$ Dipartimento di Chimica Inorganica, Metallorganica e Analitica,Università di Milano, Centro CIMAINA, UdR di Milano INSTM and Centro ISTM-CNR, Via Venezian 21, 20133Milano, Italy; ${ }^{b}$ Dipartimento di Scienze

Chimiche e Ambientali, Università dell'Insubria, Via Valleggio 11, 22100 Como, Italy; ${ }^{c}$ Dipartimento di Chimica Strutturale e Stereochimica Inorganica and Centro ISTM-CNR, via Venezian 21, 20133 Milano Italy;

${ }^{d}$ Dipartimento di Chimica Università di Firenze and UdR di Firenze INSTM, via della Lastruccia 3, 50019 Sesto Fiorentino (FI), Italy

E-mails: elena.cariati@unimi.it, piero.macchi@unimi.it, dante.gatteschi@unifi.it
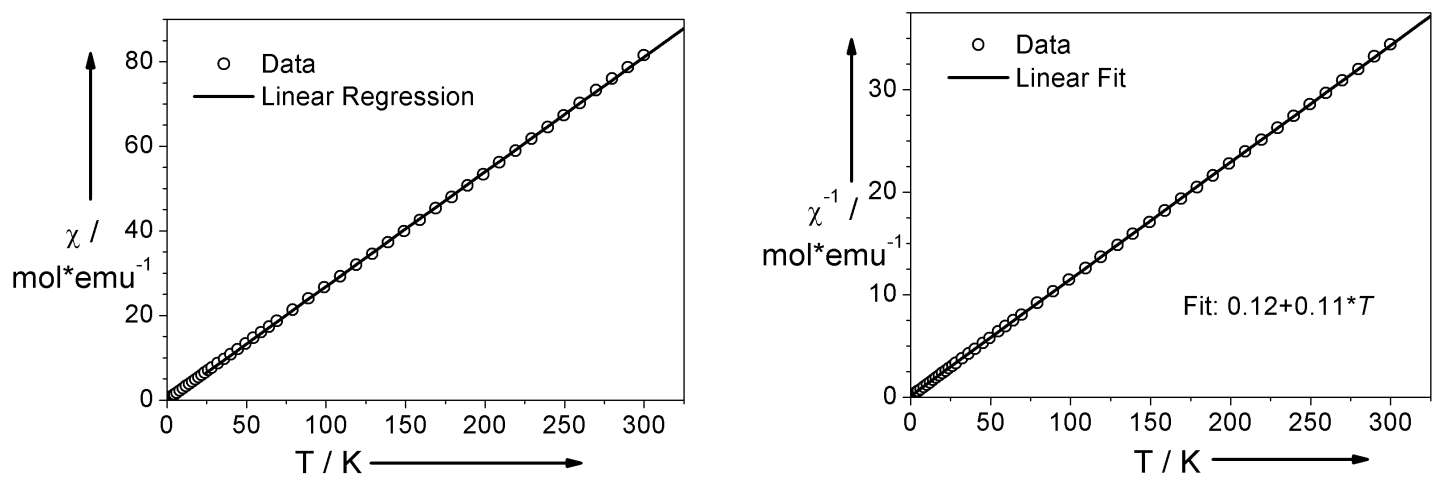

Curie-Weiss low plots of [DAMS $]_{4}\left[\mathrm{Fe}_{2} \mathrm{Mn}\left(\mathrm{C}_{2} \mathrm{O}_{4}\right)_{6}\right] \cdot 2 \mathrm{DAMBA} \cdot 2 \mathrm{H}_{2} \mathrm{O}$ (left) and $[\mathrm{DAMS}]_{4}\left[\mathrm{Cr}_{2} \mathrm{Mn}\left(\mathrm{C}_{2} \mathrm{O}_{4}\right)_{6}\right] \cdot 2 \mathrm{DAMBA} \cdot 2 \mathrm{H}_{2} \mathrm{O}$ (right) 